\title{
Wrapping and dispersion of multiwalled carbon nanotubes improves electrical conductivity of protein-nanotube composite biomaterials
}

\author{
Christopher M. Voge,, Jeremy Johns, ${ }^{1}$ Mekhala Raghavan, ${ }^{1,2}$ Michael D. Morris, ${ }^{2}$ \\ Jan P. Stegemann ${ }^{1}$ \\ ${ }^{1}$ Department of Biomedical Engineering, University of Michigan, Ann Arbor, 1101 Beal Ave., Ann Arbor, Michigan 48109 \\ ${ }^{2}$ Department of Chemistry, University of Michigan, Ann Arbor, 930 N. University Ave., Ann Arbor, Michigan 48108
}

Received 20 December 2011; revised 17 April 2012; accepted 24 May 2012

Published online 3 August 2012 in Wiley Online Library (wileyonlinelibrary.com). DOI: 10.1002/jbm.a.34310

\begin{abstract}
Composites of extracellular matrix proteins reinforced with carbon nanotubes have the potential to be used as conductive biopolymers in a variety of biomaterial applications. In this study, the effect of functionalization and polymer wrapping on the dispersion of multiwalled carbon nanotubes (MWCNT) in aqueous media was examined. Carboxylated MWCNT were wrapped in either Pluronic ${ }^{\circledR}$ F127 or gelatin. Raman spectroscopy and X-ray photoelectron spectroscopy showed that covalent functionalization of the pristine nanotubes disrupted the carbon lattice and added carboxyl groups. Polymer and gelatin wrapping resulted in increased surface adsorbed oxygen and nitrogen, respectively. Wrapping also markedly increased the stability of MWCNT suspensions in water as measured by settling time and zeta potential, with Pluronic ${ }^{\circledR}$-wrapped nanotubes showing the greatest effect. Treated MWCNT were used to make
\end{abstract}

3D collagen-fibrin-MWCNT composite materials. Carboxylated MWCNT resulted in a decrease in construct impedance by an order of magnitude, and wrapping with Pluronic ${ }^{\circledR}$ resulted in a further order of magnitude decrease. Functionalization and wrapping also were associated with maintenance of fibroblast function within protein-MWCNT materials. These data show that increased dispersion of nanotubes in protein-MWCNT composites leads to higher conductivity and improved cytocompatibility. Understanding how nanotubes interact with biological systems is important in enabling the development of new biomedical technologies. (c) 2012 Wiley Periodicals, Inc. J Biomed Mater Res Part A: 101A: 231-238, 2013.

Key Words: carbon nanotubes, composite, collagen, fibroblast, nanoparticle, pluronic

How to cite this article: Voge CM, Johns J, Raghavan M, Morris MD, Stegemann JP. 2013. Wrapping and dispersion of multiwalled carbon nanotubes improves electrical conductivity of protein-nanotube composite biomaterials. J Biomed Mater Res Part A 2013:101A:231-238.

\section{INTRODUCTION}

The last decade has seen a great deal of activity in the use of nanoparticles to augment the properties of a variety of materials, including biomaterials. ${ }^{1,2}$ Among these, composites of polymers and carbon nanotubes (CNT) have been pursued vigorously because of the remarkable properties of CNT (strength, flexibility, electrical conductivity, high aspect ratio) that make them attractive as a reinforcing phase., However, in many cases, the theoretical benefits of CNTpolymer composites have not been realized, largely because the properties of CNT are unique, and their very small size makes it difficult to control their spatial organization once they have been fabricated. Despite these hurdles, it is clear that CNT composites have high potential in many applications. A key to unlocking their potential is to understand and control the interactions between CNT and their surrounding environment.

Conductive biomaterials are desirable for a range of applications, including neural scaffolds, biosensors, and device coatings. A number of polymer-based conductive materials have been investigated for these applications, including polyethylenedioxythiophene, ${ }^{5}$ polypyrrole, ${ }^{6}$ polyaniline, $^{7}$ and other redox-based conductors. More recently, there have been reports of combining natural polymers (i.e., proteins and polysaccharides) with CNT to create composites that exhibit electrical conductivity. ${ }^{8,9}$ Natural extracellular matrix proteins such as collagen, elastin, and laminin have several potential advantages over synthetic polymers, including higher levels of biocompatibility and bioactivity. CNT are promising reinforcing phases for these materials because of their ability to conduct electronically, while also being more chemically inert and stable compared to synthetic conductive polymers. ${ }^{10} \mathrm{CNT}$ also provide the opportunity for custom chemical functionalization based on the application and environment.

Our previous work demonstrated that carboxylated single-walled carbon nanotubes (SWCNT) can improve the electrical properties of collagen hydrogels. ${ }^{11}$ The additional

Correspondence to: J. P. Stegemann; e-mail: jpsteg@umich.edu 


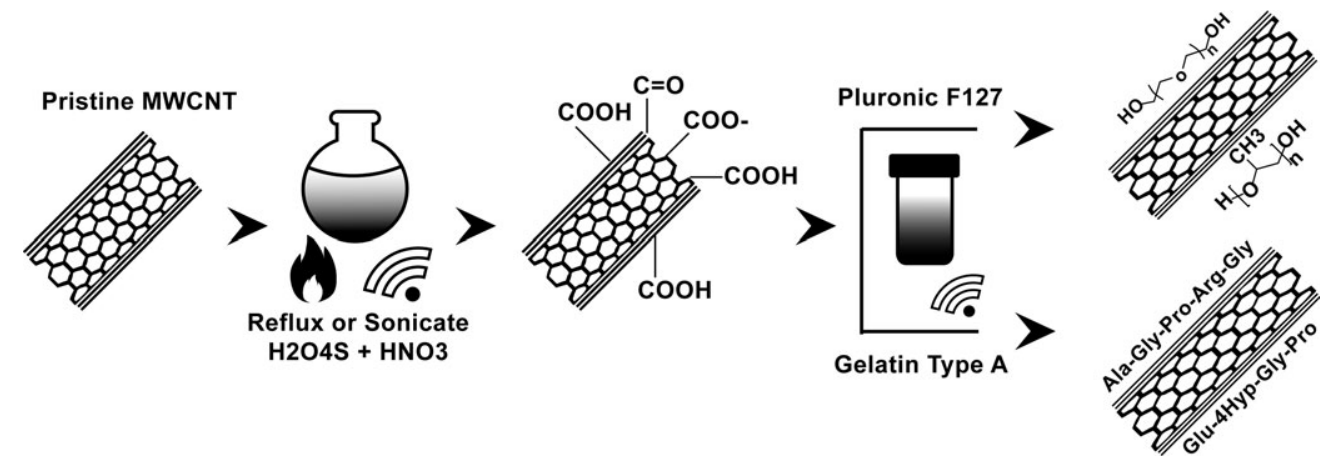

FIGURE 1. Schematic of MWCNT functionalization and wrapping process. Pristine MWCNT were first carboxylated by acid reflux or sonication and then wrapped with either Pluronic ${ }^{\circledR}$ F127 or gelatin in a sonication bath.

incorporation of fibrin into these hydrogels improved compaction and assisted with cell-mediated alignment of SWCNT within the matrix. ${ }^{12}$ Although these studies showed promise in creating electrically conductive biopolymers, it was determined that electrical percolation had not been achieved in these materials. For this reason, we have focused on achieving increased dispersion of CNT within protein matrices to maximize electrically conductive paths within the material. One of the most effective methods to improve CNT dispersion in aqueous solutions is the addition of polar chemical groups to the surface, either covalently or using noncovalent wrapping techniques. Covalent attachment of polar groups increases solubility and can also serve to remove potentially toxic metallic impurities from CNT preparations ${ }^{13,14}$; however, such modification is destructive to the electrical and mechanical properties of the CNT lattice. ${ }^{15}$ Noncovalent coating of CNT with ionic surfactants, polymers, and oligopeptides has also been used as a strategy to increase dispersion. ${ }^{16}$ Such wrapping techniques benefit from being nondestructive and efficient, ${ }^{17}$ but many of the best dispersants are also toxic to living cells.

The current study extends our previous work to functionalized multiwalled carbon nanotubes (MWCNT) and uses wrapping strategies to increase dispersion in aqueous media. MWCNT have advantages as an electrically conductive filler in protein composites because defects that are caused by functionalization affect only the outer wall of the MWCNT, leaving the inner concentric walls intact and therefore allowing uninterrupted electron flow. The wrapping materials examined are Pluronic ${ }^{\circledR}$ F127 (a commercially available polyethylene oxide (PEO)-polypropylene oxide (PPO) copolymer) and gelatin (a denatured collagen peptide with $\mathrm{MW} \sim 100$ $\mathrm{kDa}$ ). Pluronic ${ }^{\circledR}$ has been shown previously to be effective at dispersing CNT in solution. ${ }^{18}$ Gelatin was chosen based on previous work demonstrating that high-molecular weight proteins effectively wrap CNT. ${ }^{19}$ Both of these dispersing agents are compatible with cells at low concentrations. ${ }^{20}$ The overall goal of this work is to obtain improved dispersion of MWCNT in aqueous media for the purpose of creating 3D CNT-protein composite materials that support the maintenance and function of embedded cells. Such electrically conductive biopolymer scaffolds could have utility in the fields of biosensing, neural prosthetics, and tissue engineering.

\section{MATERIALS AND METHODS}

Functionalization and wrapping of MWCNT

Figure 1 shows a schematic diagram of how nanotubes were purified, carboxylated, and wrapped. Table I shows the different treatments of MWCNT that were examined in this study, including the reflux type/time and the wrapping method. MWCNT (diameter 6-13 nm, length 2-20 $\mu \mathrm{m}$; Sigma Aldrich, St. Louis MO) were refluxed or sonicated in a 3:1 solution of $5 M$ sulfuric and nitric acid. Sonication was performed at a power level of $40 \mathrm{~W}$, and the temperature was maintained below $60^{\circ} \mathrm{C}$. The refluxing solution was maintained at $\sim 200^{\circ} \mathrm{C}$ while being stirred in a closed system. The solution was then filtered through a $0.2-\mu \mathrm{m}$ hydrophilic PTFE membrane with sterile water and neutralized using $\mathrm{NaOH}$. Both refluxing and sonication in acid serve to create defects in the carbon lattice to which carboxyl groups can attach. This treatment also purifies the preparation of MWCNT by removing any residual metallic catalyst.

Physical wrapping of MWCNT was performed using a sonication bath at $40 \mathrm{~W}$ for $1 \mathrm{~h}$. Pluronic ${ }^{\circledR}$ F127 (BASF SE, Germany) or gelatin (type A, 225 bloom; MP Biomedical, Solon, $\mathrm{OH}$ ) was dissolved in a solution of MWCNT at 0.01 wt $\%$ and then sonicated for $1 \mathrm{~h}$. The suspension was then filtered through a $0.2-\mu \mathrm{m}$ PTFE membrane and the MWCNT were resuspended in sterile water.

\section{TABLE I. MWCNT Treatment Types}

\begin{tabular}{llll}
\hline Sample & $\begin{array}{l}\text { Reflux (R) or } \\
\text { Sonication (S) }\end{array}$ & Duration & $\begin{array}{c}\text { Pluronic or } \\
\text { Gelatin }\end{array}$ \\
\hline MW & N/A & N/A & N/A \\
MW2R & $\mathrm{R}$ & $2 \mathrm{~h}$ & N/A \\
MW15S & $\mathrm{S}$ & $15 \mathrm{~s}$ & $\mathrm{~N} / \mathrm{A}$ \\
MW2S & $\mathrm{S}$ & $2 \mathrm{~h}$ & $\mathrm{~N} / \mathrm{A}$ \\
MW12S & $\mathrm{S}$ & $12 \mathrm{~h}$ & $\mathrm{~N} / \mathrm{A}$ \\
MW2R-P & $\mathrm{R}$ & $2 \mathrm{~h}$ & $\mathrm{P}$ \\
MW15S-P & $\mathrm{S}$ & $15 \mathrm{~s}$ & $\mathrm{P}$ \\
MW2S-P & $\mathrm{S}$ & $2 \mathrm{~h}$ & $\mathrm{P}$ \\
MW12S-P & $\mathrm{S}$ & $12 \mathrm{~h}$ & $\mathrm{P}$ \\
MW2R-G & $\mathrm{R}$ & $2 \mathrm{~h}$ & $\mathrm{G}$ \\
MW15S-G & $\mathrm{S}$ & $15 \mathrm{~s}$ & $\mathrm{G}$ \\
MW2S-G & $\mathrm{S}$ & $2 \mathrm{~h}$ & $\mathrm{G}$ \\
MW12S-G & $\mathrm{S}$ & $12 \mathrm{~h}$ & $\mathrm{G}$
\end{tabular}




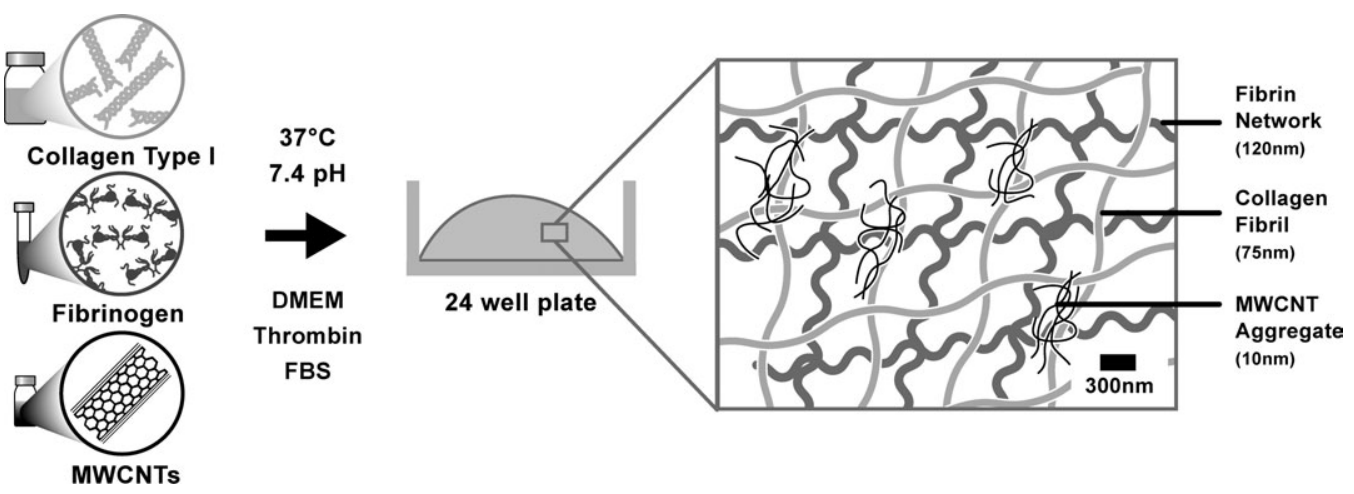

FIGURE 2. Schematic illustration of MWCNT-collagen-fibrin composite fabrication and composition. The components are mixed as solutions and turned into a hydrogel composite containing three fiber types.

\section{MWCNT characterization}

Chemical information was obtained using a Kratos Axis Ultra X-ray photospectrometer (XPS; Kratos Analytical, Japan) with a monochromatic Al source in spectrum mode operating at $\sim 10 \mathrm{~mA}$ and $\sim 15 \mathrm{kV}$. Samples were prepared by pressing the MWCNT powder into 1-mm-thick indium foil squares. A representative spectrum was obtained from multiple spots on each sample, including focused scans for oxygen and nitrogen peaks.

Raman spectra were obtained using a Raman microprobe system constructed around a Nikon E600 microscope frame (Nikon USA, Melville, New York, NY). The exciting line laser was a 400-mW, 785-nm diode laser (Invictus, Kaiser Optical Systems, Ann Arbor, Michigan). The laser power was attenuated using a neutral density filter of optical density 2.0 (Thor Labs, Newton, New Jersey) and focused on to the sample through a NIR-optimized $40 \times, 0.90$ NA objective (Fluor series, Nikon USA, Melville, New York, NY). The laser power at the sample was $\sim 1 \mathrm{~mW}$, and all measurements were taken at room temperature. An axial transmissive imaging spectrograph (HoloSpec, Kaiser Optical Systems) with 25- $\mu \mathrm{m}$ entrance slit (approximately 3-4 $\mathrm{cm}^{-1}$ resolution) and a $1024 \times 256$ pixel deep depletion chargecoupled device detector (Model DU 401-BR-DD, Andor Technology, South Windsor, CT) were used to disperse and record the Raman spectrum.

Powder samples were pressed into indium foil and Raman spectra were collected at different spots on each sample at acquisition times of 30-60 s. Spectral data analysis was performed using Matlab (MathWorks, Natick, MA) and GRAMS/AI 7.01 (Thermo Galactic, Waltham, MA) software. Spectra over the range of $800-1800 \mathrm{~cm}^{-1}$ were preprocessed to correct for image curvature, remove detectorgenerated artifacts (spikes), subtract detector dark current, and perform white light correction. Raman peaks positions and intensities were analyzed by a Lorentzian fit of the spectra. ${ }^{21}$ Statistical tests were performed on band positions and intensity ratios using two-tailed unpaired $t$ tests to compare the effect of functionalization on MWCNT. A value of $p<0.05$ was considered significant.

The zeta potential of MWCNT suspensions was characterized using the Helmholtz-Smoluchowski equation on a
Brookhaven 90plus BI-Zeta Particle Sizer (Brookhaven Instrument Corporation, New York, NY) and a palladium electrode cell. MWCNT samples were dispersed at a concentration of $0.05 \mathrm{mg} / \mathrm{mL}$ in de-ionized water at $\mathrm{pH}$ 7.4. The settling time of a subset of MWNCT samples was also characterized to qualitatively observe the effect of functionalization and wrapping. Nanotubes were dispersed in de-ionized water in 10 $\mathrm{mL}$ sample tubes, and the suspension was allowed to sit undisturbed for $12 \mathrm{~h}$. Digital images were taken immediately after dispersion and at the end of the experiment.

\section{Preparation and characterization of MWCNT-collagen-fibrin composites}

The components and process for making 3D MWCNT-collagen-fibrin composite materials is shown schematically in Figure 2. Bovine collagen type I (MP Biomedicals, Solon, $\mathrm{OH}$ ) was dissolved in cold $0.2 \mathrm{M}$ acetic acid at $4.0 \mathrm{mg} / \mathrm{mL}$. Bovine fibrinogen (Sigma Chemical, St. Louis, MO) was dissolved in Dulbecco's modified eagle medium containing 4.0 $\mathrm{mg} / \mathrm{mL} \varepsilon$-aminocaproic acid (DMEM, Invitrogen, Carlsbad, CA) at $4.0 \mathrm{mg} / \mathrm{mL}$. These matrix solutions were combined at a 1:1 ratio, and the appropriate volumes of fetal bovine serum (10\%, Invitrogen), 5× DMEM solution, and MWCNT suspended in sterile de-ionized water were added. Gelation of the collagen and fibrin phases was initiated by neutralization with $0.1 \mathrm{M} \mathrm{NaOH}$, addition of thrombin, and increasing the temperature to $37^{\circ} \mathrm{C}$. The volumes of the components were calculated to produce 3D MWCNT-protein constructs containing a final concentration of $1.0 \mathrm{mg} / \mathrm{mL}$ collagen and $1.0 \mathrm{mg} / \mathrm{mL}$ fibrin. Control constructs (CNTR) that did not contain MWCNT were also fabricated for comparison.

For impedance measurements, acellular constructs were produced with a final MWCNT concentration of $0.3 \mathrm{mg} / \mathrm{mL}$ and air dried into 1-cm diameter circular thin sheets. As shown in Figure 3, micro-wire electrodes were attached to the composite material using silver epoxy (Electron Microscopy Sciences, Hatfield, PA) and electrochemical impedance measurements were obtained using a potentiostat (Autolab, Utrecht, Netherlands). Impedance values were measured in triplicate from 10 to $10,000 \mathrm{~Hz}$ using an excitation signal of $25 \mathrm{mV}$. MATLAB was used to analyze impedance spectra and to generate Bode and Nyquist plots. 
A
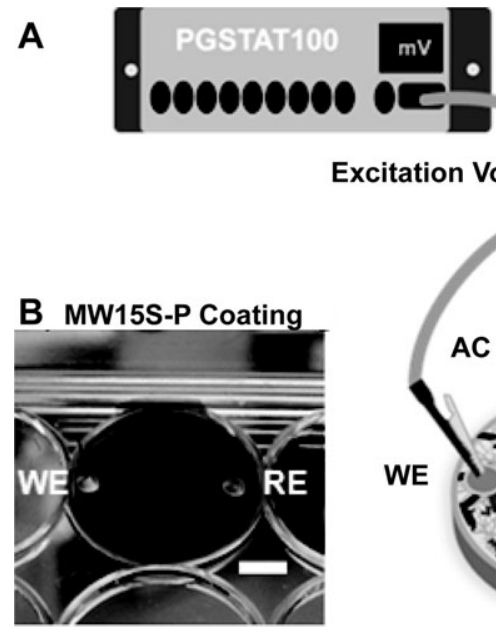

Excitation Voltage (V)

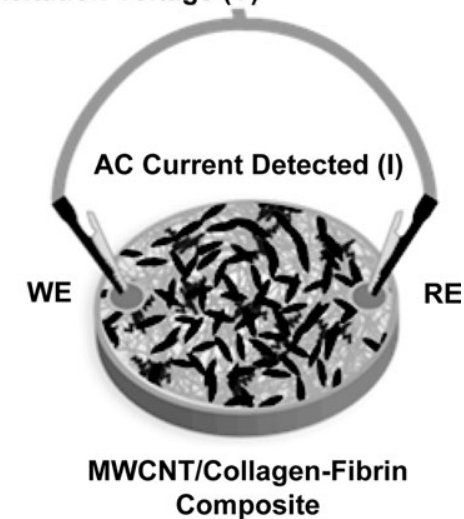

FIGURE 3. (A) Schematic of two-point bioimpedance testing configuration and (B) image of MW15S-P protein composite prepared for testing. Scale bar represents $3 \mathrm{~mm}$.

Biocompatibility experiments were performed using 100 $\mu \mathrm{L}$ collagen-fibrin constructs seeded with human neonatal dermal fibroblasts (Invitrogen) at a concentration of $0.5 \times$ $10^{6}$ cells $/ \mathrm{mL}$. Importantly, fibroblasts were added directly to the MWCNT-protein solution before initiation of gelation so that the cells were embedded and homogenously distributed throughout the construct matrix. Fibroblast-seeded constructs were cultured at $37^{\circ} \mathrm{C}$ and fed every other day with DMEM containing 10\% FBS and 1\% penicillin streptomycin L-glutamine. For cell studies, MWCNT concentrations of $0.6,0.3,0.15$, and $0.075 \mathrm{mg} / \mathrm{mL}$ were tested. A colorimetric tetrazolium salt assay based on the conversion of $3^{\prime}-\{1$ [(phenylamino)-carbonyl]-3,4-tetrazolium\}-bis (4-methoxy-6nitro) benzene-sulfonic acid hydrate (XTT; Roche Chemicals, Indianapolis, IN) was used to quantify the metabolic activity of fibroblasts in gels at days 1, 3, and 7. Constructs were incubated with $200 \mu \mathrm{L}$ of XTT reagent and $300 \mu \mathrm{L}$ of cell media for $12 \mathrm{~h}$. The supernatant was removed from the sample well, and absorbance of samples was recorded in a 96-well plate at $550 \mathrm{~nm}$. The degree of XTT conversion is indicated by the degree of color change, which therefore is a reflection of the metabolic activity of the cells embedded in the constructs.

\section{RESULTS AND DISCUSSION}

\section{Functionalization and wrapping improves MWCNT} dispersion and stability

Raman spectroscopy data for pristine and functionalized MWCNT are shown in Figure 4. Both representative curves exhibited the disorder-induced $\mathrm{D}$ and $\mathrm{D}^{\prime}$ bands $(\sim 1310$ $\mathrm{cm}^{-1}$ and $\sim 1600 \mathrm{~cm}^{-1}$, respectively) and the graphitic (G) band $\left(1580 \mathrm{~cm}^{-1}\right)$ characteristic of CNT spectra. The D and $\mathrm{D}^{\prime}$ bands are double resonance bands that serve as a measure of structural disorder. The $\mathrm{G}$ band results from the tangential $\mathrm{C}-\mathrm{C}$ stretching vibrations both longitudinally and transversally on the CNT axis. ${ }^{22}$ It should be noted that the
D band is dependent on the excitation wavelength for MWCNT. At a wavelength of $785 \mathrm{~nm}$, the D band is emphasized more than the $\mathrm{G}$ band. In addition, the $\mathrm{D}$ band position is centered at around $1300 \mathrm{~cm}^{-1}$ rather than at 1357 $\mathrm{cm}^{-1}$ at a wavelength of $488 \mathrm{~nm}^{23}$

Table II shows that functionalization caused a significant shift to higher wavenumbers in the $D, G$, and $D^{\prime}$ bands of the spectrum, indicating that a chemical species had been absorbed or was interacting with the outer carbon lattice. The ratio of the intensities of the $D$ to $G$ bands can reveal information about the extent of damage in the pristine carbon lattice caused by functionalization. ${ }^{24}$ Acid-treated samples had increased D/G intensity ratios compared with pristine samples, indicating more disordered carbon (defects in NT walls). However, even pristine MWCNT had a higher $D / G$ ratio than would be expected for completely undamaged nanotubes, suggesting that the source MWCNT contained residual defects from the manufacturing or purification process. Damage to the carbon lattice could negatively affect the electrical conductivity quality of the nanotubes by interrupting the flow of electrons. A significant shift to higher wavenumbers in the $\mathrm{D}, \mathrm{D}^{\prime}$, and $\mathrm{G}$ peaks was also observed in the Pluronic ${ }^{\circledR}$ - and gelatin-coated samples (Table II), indicating that absorption of polar species occurred on the nanotube surface. ${ }^{25}$ These shifts were distinct from acid-treated samples, suggesting that a different interfacial interaction is occurring between the polymer wrapping and the outer carbon lattice. However, the $D / G$ intensity ratios were not significantly different from the pristine or MW2R samples, indicating that the wrapping process did not damage the lattice further.

Examination of the surface chemistry of MWCNT powders using XPS provided further insight into the specific chemical effects of functionalization and wrapping. XPS data are shown in Figure 5. The representative curves in Figure $5(\mathrm{~A})$ show the characteristic carbon peak $(\sim 280 \mathrm{eV})$ expected from MWCNT, and the spectra of functionalized samples show peaks associated with atomic nitrogen $(\sim 400$ $\mathrm{eV}$ ) and oxygen ( $530 \mathrm{eV})$. Figure $5(\mathrm{~B})$ shows that pristine MWCNT contained $\sim 2 \%$ atomic oxygen and that carboxylation increased this value to between 5 and $10 \%$. Wrapping of MWCNT with Pluronic further increased surface oxygen

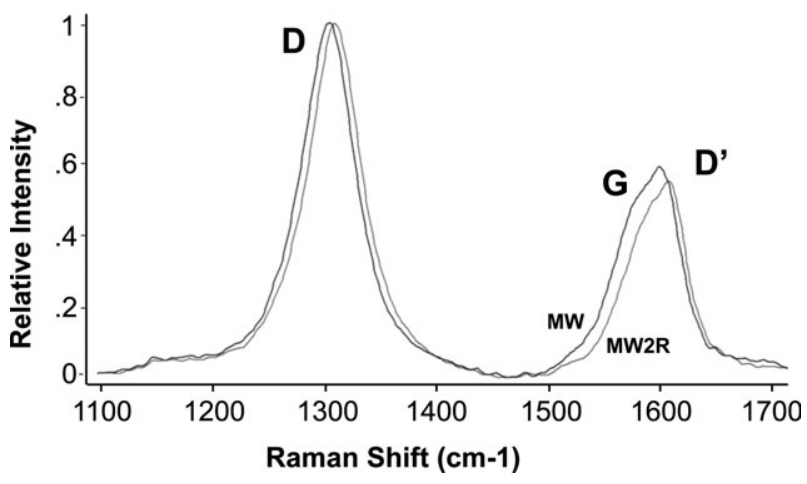

FIGURE 4. Representative Raman spectra of pristine and functionalized MWCNT showing characteristic disorder bands (D) and ( $\left.D^{\prime}\right)$ and graphitic (G) band. 
TABLE II. Raman Shifts (Mean \pm SD) and Changes in Ratio of D/G Intensity Caused by Functionalization of MWCNT

\begin{tabular}{lcccr}
\hline & D Band $\left(\mathrm{cm}^{-1}\right)$ & G Band $\left(\mathrm{cm}^{-1}\right)$ & ${\text { D Band }\left(\mathrm{cm}^{-1}\right)}$ & ID/IG \\
\hline MW & $1304.6 \pm 0.8$ & $1574.0 \pm 1.0$ & $1603.7 \pm 1.0$ & $3.41 \pm 0.27$ \\
MW2R & $1309.0 \pm 1.1^{\mathrm{a}}$ & $1579.6 \pm 1.2^{\mathrm{a}}$ & $1607.9 \pm 0.9^{\mathrm{a}}$ & $3.62 \pm 0.35$ \\
MW15S & $1308.1 \pm 1.2^{\mathrm{a}}$ & $1576.2 \pm 1.1^{\mathrm{a}}$ & $1607.0 \pm 1.2^{\mathrm{a}}$ & $3.86 \pm 0.26^{\mathrm{a}}$ \\
MW2S & $1307.9 \pm 0.2^{\mathrm{a}}$ & $1576.8 \pm 0.4^{\mathrm{a}}$ & $1606.8 \pm 0.5^{\mathrm{a}}$ & $3.86 \pm 0.11^{\mathrm{a}}$ \\
MW12S & $1307.2 \pm 1.0^{\mathrm{a}}$ & $1576.4 \pm 1.1^{\mathrm{a}}$ & $1606.2 \pm 1.0^{\mathrm{a}}$ & $3.76 \pm 0.15^{\mathrm{a}}$ \\
MW2R-P & $1309.4 \pm 0.1^{\mathrm{a}}$ & $1581.8 \pm 0.4^{\mathrm{a}}$ & $1609.7 \pm 0.3^{\mathrm{a}}$ & $3.22 \pm 0.11$ \\
MW2R-G & $1310.8 \pm 0.2^{\mathrm{a}}$ & $1581.8 \pm 0.5^{\mathrm{a}}$ & $1609.9 \pm 0.3^{\mathrm{a}}$ & $3.19 \pm 0.05$ \\
\hline
\end{tabular}

a Statistical difference from unfunctionalized MW controls at $p<0.05$.

to between 20 and 28\%, while gelatin wrapping had a similar effect. Nitrogen was not detected on pristine MWCNT and was present at low levels (1-2\%) on functionalized and Pluronic ${ }^{\circledR}$-wrapped MWCNT. However, wrapping with gelatin increased nitrogen surface density to around 10\%, which reflects the presence of amine groups from the peptide adsorbed to the nanotube surface. These XPS data show that the surface of the MWCNT was oxidized by chemical functionalization and verify the adsorption of Pluronic ${ }^{\circledR}$ and gelatin to the nanotube surface.

Changes in MWCNT surface chemistry can markedly affect how they interact with their environment. Dispersion of MWCNT in aqueous medium was affected by the degree and type of functionalization and wrapping, as shown in Figure 6. Settling time experiments of a subset of treatments [Fig. 6(A)] showed that carboxylated samples remained better dispersed over a 12 -h period, relative to pristine MWCNT controls. Pluronic ${ }^{\circledR}-$ and gelatin-wrapped MWCNT exhibited the lowest degree of settling, with Pluronic ${ }^{\circledR}$-wrapped tubes being the most stable. These results were validated using zeta potential measurements [Fig. 6(B)], which reflects colloidal stability of the MWCNT suspension. ${ }^{26} \mathrm{~A}$ high absolute value of the zeta potential is generally indicative of resistance to particle aggregation and a correspondingly stable solution. This assay revealed that the zeta potential was negative and that Pluronic ${ }^{\circledR}$ wrapping caused the greatest increase in potential. Pluronic ${ }^{\circledR}$ surfactants are used industrially as dispersants and are designed to increase the aqueous solubility of hydrophobic materials. This family of block copolymers has been used in the pharmaceutical and drug delivery applications and is well suited for biological applications because of its cytocompatibility at low concentrations. ${ }^{27,28}$ The effect of gelatin was more moderate, whereas functionalization without wrapping had essentially no effect on the measured zeta potential.

Increased nanotube dispersion reduces impedance of MWCNT-protein composite materials

Figure 7 shows bioimpedance spectroscopy data for MWCNT-collagen-fibrin films, relative to pure collagenfibrin controls (CNTR). Impedance data were measured over a spectrum of frequencies from 10 to $10,000 \mathrm{~Hz}$, and the magnitude and phase of the output were measured. The impedance measurements shown in Figure 7(A) were evaluated at a frequency of $1000 \mathrm{~Hz}$. This value was chosen as the midrange of the biologically relevant frequency associated with neuronal activity. Inclusion of pristine MWCNT in collagen-fibrin constructs reduced the impedance by a factor of two compared with pure collagen-fibrin constructs [Fig. 7(A)]. Carboxylated and gelatin-wrapped MWCNT reduced collagen-fibrin construct impedance by an order of magnitude relative to control and pristine MWCNT samples. Pluronic ${ }^{\circledR}$-wrapped MWCNT showed significantly smaller impedances compared with all other MWCNT samples, with MW12S-P exhibiting a two order of magnitude reduction in impedance.

The Bode [Fig. 7(B)] and Nyquist [Fig. 7(C)] plots were generated from the frequency response data for the sample with the lowest impedance, MW12S-P. The Bode plot shows
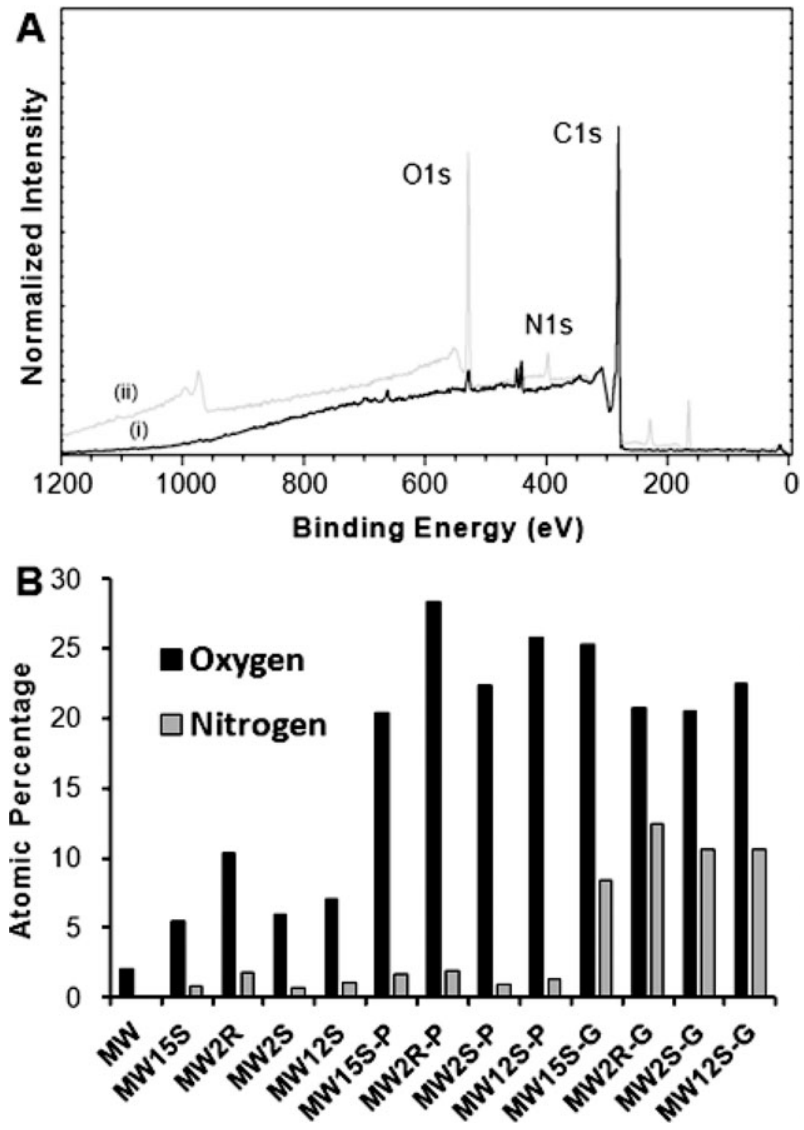

FIGURE 5. (A) Representative XPS spectra of (i) pristine MW and (ii) MW2R showing characteristic peaks and addition of $\mathrm{O}$ and $\mathrm{N}$ groups. (B) Aggregate data obtained from XPS measurements on treated MWCNT samples. 

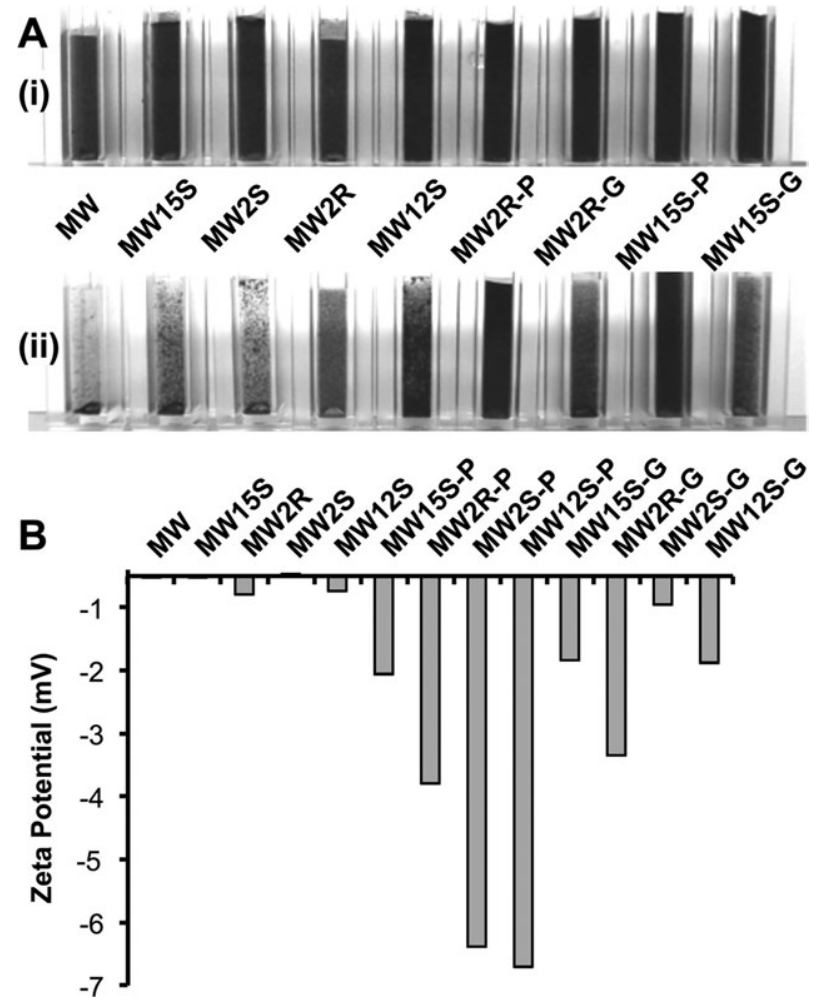

FIGURE 6. (A) Images of settling experiments at (i) $t=0$ and (ii) $t=$ $12 \mathrm{~h}$ for selected functionalized and wrapped MWCNT. (B) Zeta potential values for treated MWCNT.

that material impedance was dependent on frequency, suggesting that full electrical percolation across the sample had not been achieved in the composites. If the electric current was traveling only through the CNT phase, then a more constant impedance level over the frequency spectrum would be expected. The Nyquist plot shows the real versus imaginary component of the impedance and can be used to compare how the complex MWCNT-protein system behaved relative to classical electrical models. The angle of the curve in Figure $7(\mathrm{C})$ is approximately $45-55^{\circ}$, indicating that the composite material exhibits Warburg impedance, which reflects a diffusive element in the circuit and suggests that percolation was not achieved.

The bioimpedance spectroscopy data provide insight into how MWCNT affect the electrical properties of 3D protein matrices and show the effects of nanotube dispersion on these properties. In general, MWCNT-protein materials that were made with more highly dispersed nanotubes exhibited lower impedances. The most dielectric samples contained MWCNT with zeta potentials close to zero, indicating poor dispersion and stability in aqueous solution. The formulations of MWCNT that were most stable in solution, which were both functionalized and wrapped with Pluronic $^{\circledR}$, showed a two order of magnitude decrease in electrical impedance relative to matrices containing pristine nanotubes. However, even with markedly increased dispersion of MWCNT, the impedance analysis suggested that a fully percolating electrical network phase did not yet exist in the hydrogels. ${ }^{29,30}$ Other groups that have used CNT as fillers in synthetic polymer materials have reported lower impedances, including the achievement of electrical percolation. $^{31,32}$ It should be noted, however, that these materials are made using efficient but toxic dispersing agents, as well as processing temperatures and techniques that would not allow the inclusion of cells in the material. These data illustrate the challenge in creating electrically conductive protein-based biomaterials with cells embedded directly in the matrix at the time of fabrication.

\section{Cellular activity in 3D protein-MWCNT composites}

The XTT assay quantifies mitochondrial activity of cells, which has been used as a reflection of overall cellular activity and viability. ${ }^{33}$ An important advantage of the XTT dye is that the conversion product is water soluble. It therefore can be used to assess cell viability in 3D protein matrices.
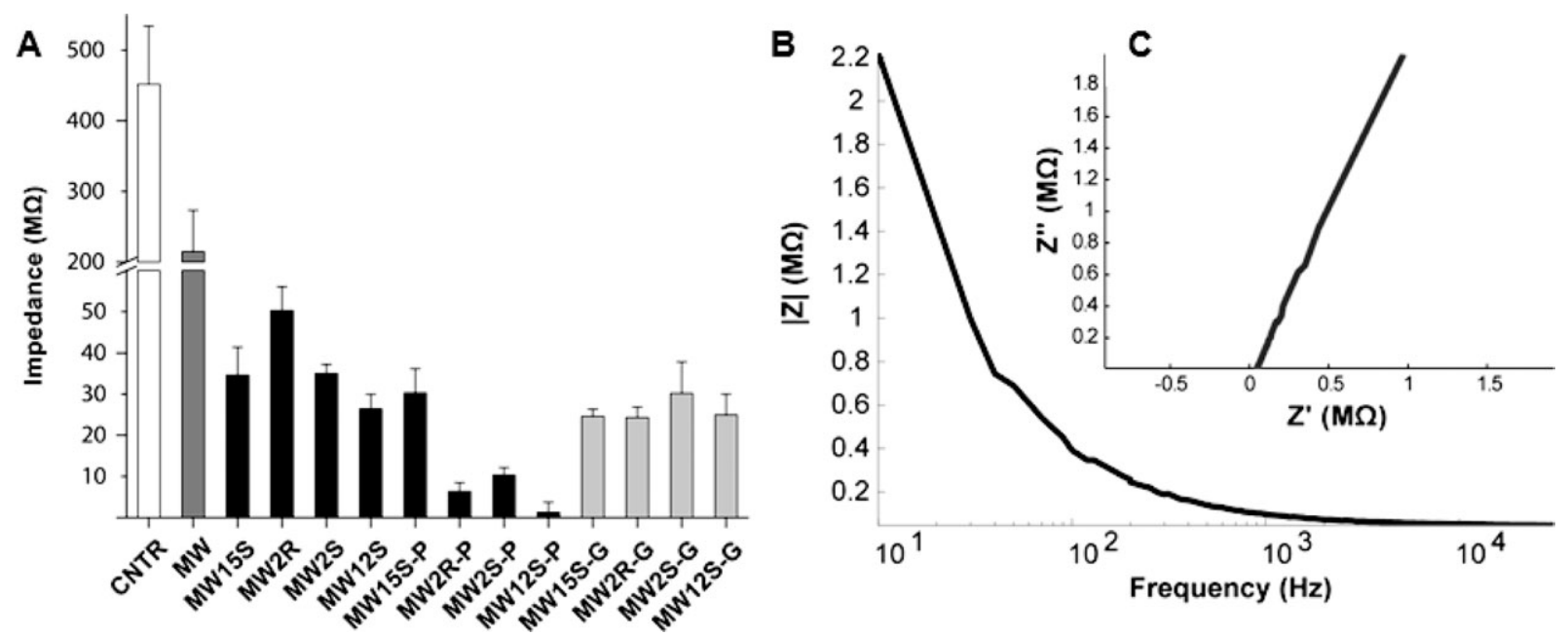

FIGURE 7. (A) Impedance values at $1 \mathrm{kHz}$ for MWCNT-protein composite materials as a function of functionalization and wrapping method. CNTR indicates collagen-fibrin materials without nanotubes. (B) Bode magnitude plot for MW12S-P and (C) Nyquist plot for MW12S-P $\left({ }^{*} p<0.05\right)$. 


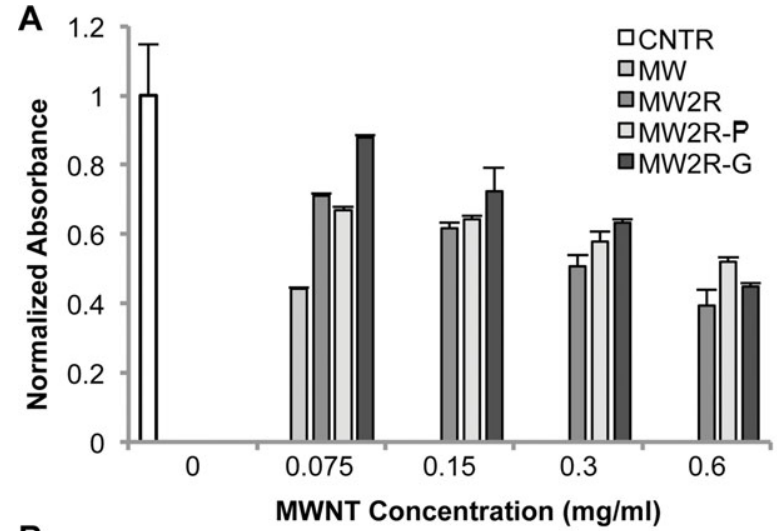

B

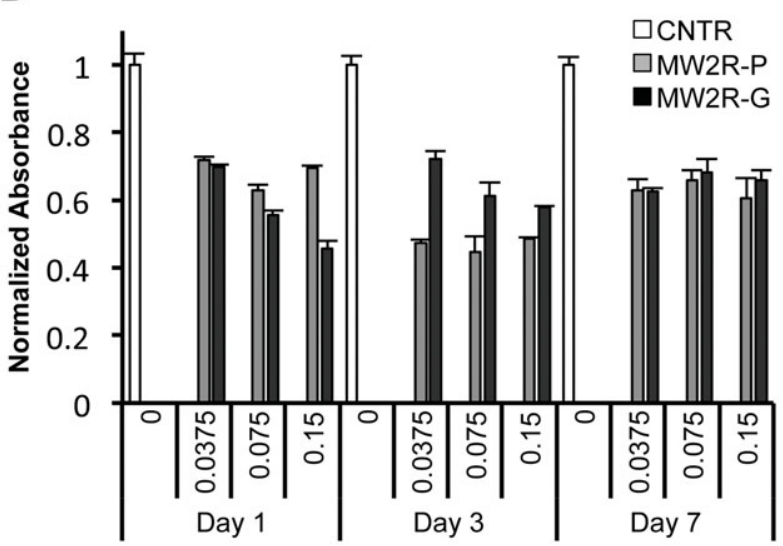

MWNT Concentration $(\mathrm{mg} / \mathrm{ml})$
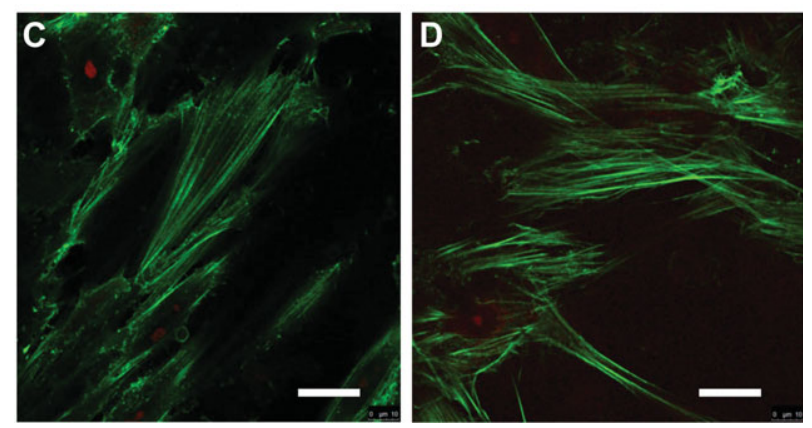

FIGURE 8. (A) Metabolic activity of fibroblasts in MWCNT-protein composites as a function of concentration after $24 \mathrm{~h}$. CNTR indicates collagen-fibrin constructs without nanotubes. (B) Metabolic activity of fibroblasts in MWCNT-protein composites as a function of concentration and time in culture. Confocal microscopy image of fibroblasts embedded in control (C) and $0.075 \mathrm{mg} / \mathrm{mL}$ MW2R constructs (D) at day 3 in culture show the cytoskeleton of the cells stained green. Scale bar represents $20 \mu \mathrm{m}$. $\left(^{*} p<0.05\right)$. [Color figure can be viewed in the online issue, which is available at wileyonlinelibrary.com.]

In addition, XTT can be used on nanotube-containing samples, which have been shown to interfere with readings in other insoluble tetrazolium salt-based assays. ${ }^{34}$ Figure 8 shows the metabolic activity and morphology of human dermal fibroblast cells cultured from 1 to 7 days in MWCNTcollagen-fibrin constructs.

Figure 8(A) shows metabolic activity as a function of nanotube concentration in 3D MWCNT-protein constructs at day 1 of culture. Pristine MWCNT caused a marked decline in metabolic activity to $\sim 45 \%$ of controls at even the lowest nanotube concentration $(0.075 \mathrm{mg} / \mathrm{mL})$. These unfunctionalized nanotubes were not evaluated at higher concentrations because they aggregated in solution, which resulted in impaired gel formation. Functionalized (MW2R) and wrapped (MW2R-P and MW2R-G) nanotubes caused a dosedependent decrease in cell metabolic activity. However, both functionalization and wrapping improved the cytocompatibility of MWCNT, with Pluronic ${ }^{\circledR}$ - and gelatin-wrapped nanotubes resulting in the highest cellular activity. At $0.075 \mathrm{mg} /$ $\mathrm{mL}$, both the functionalized and wrapped nanotubes resulted in significantly greater metabolic activity, relative to pristine MWCT $(p<0.05)$. In general, at concentrations of $0.15 \mathrm{mg} / \mathrm{mL}$ and below, the treated MWCNT maintained cell metabolic activity between $\sim 60-90 \%$ of control, whereas at the highest concentration of $0.6 \mathrm{mg} / \mathrm{mL}$, the activity decreased to $\sim 40-50 \%$ of control.

Pluronic ${ }^{\circledR}$ - and gelatin-wrapped MWCNT were further evaluated for cytocompatibility over time at lower concentrations, as shown in Figure 8(B). In general, cell metabolic activity stayed approximately constant between the 1-day and 7-day time points, relative to control. Cellular activity in the MW2R-P constructs dipped slightly at day 3, but recovered by day 7. The gelatin-wrapped nanotubes showed a modest dose dependence at days 1 and 3, although this effect was not observed at day 7. At day 3, the gelatinwrapped nanotubes exhibited increased cell metabolic activity compared with Pluronic ${ }^{\circledR}$-wrapped nanotubes $(p<$ 0.05). Overall, the effect of MWCNT concentration was not as evident at these lower concentrations.

The morphology of fibroblasts embedded in pure collagen-fibrin gels [Fig. 8(C)] and MW2R composites [Fig. 8(D)] was evaluated at day 3 by staining the cytoskeleton and then imaging 3D samples using confocal microscopy. Cells in pure collagen-fibrin gels showed typical spread morphology indicative of extensive interactions with the surrounding extracellular matrix. Cells in MWCNT containing composites exhibited a similar morphology, suggesting that cell-matrix interactions were not disrupted by the presence of nanotubes. Both SWCNT and MWCNT have been shown to have potential negative effects on cell health. ${ }^{35}$ In the case of MWCNT, increased aggregation has been linked to cytotoxicity, ${ }^{36}$ although this effect was not evident in the present study. The cytocompatibility of CNT is not well understood, and there is as yet little consensus on what features of nanotubes affect their interaction with cells, although it is clear that these effects depend on the type of nanotube, dose, and mode of administration. An improved understanding of these effects is needed to use CNT composite materials for biomedical applications.

\section{CONCLUSIONS}

This study demonstrated that both functionalization and wrapping of CNT have a positive effect on the dispersion of MWCNT in aqueous solutions. These treatments reduce the hydrophobicity of the nanotubes, allowing them to form more stable solutions in water. This property is important in creating protein-based materials that incorporate 
MWCNT, because organic solvents typically denature the protein phase. Increased dispersion was associated with lower electrical impedance in MWCNT-protein materials, with PEO-PPO surfactant (Pluronic ${ }^{\circledR}$ F127) showing the most beneficial effect. However, our analysis suggested that the electrical percolation threshold had not been met in these materials. The presence of MWCNT in cell-seeded 3D MWCNT-protein matrices resulted in a general decrease in fibroblast cell metabolic function; however, polymer wrapping was associated with maintenance of cell metabolic function in matrices at low nanotube concentrations. Knowledge of how CNT interact with biological systems is important to enable the development of new materials that can be used for biomedical devices or tissue regeneration.

\section{REFERENCES}

1. Farokhzad OC, Langer R. Impact of nanotechnology on drug delivery. ACS Nano 2009;3:16-20.

2. Martin CR, Kohli P. The emerging field of nanotube biotechnology. Nat Rev Drug Discov 2003;2:29-37.

3. Moniruzzaman M, Winey KI. Polymer nanocomposites containing carbon nanotubes. Macromolecules 2006;39:5194-5205.

4. Coleman JN, Khan U, Gun'ko YK. Mechanical reinforcement of polymers using carbon nanotubes. Adv Mater 2006;18:689-706.

5. Luo SC, Xie H, Chen N, Yu H-hua. Trinity DNA detection platform by ultrasmooth and functionalized PEDOT biointerfaces. ACS App Mater Inf 2009;1:1414-1419.

6. Dong H, Cao X, Li CM. Functionalized polypyrrole film: Synthesis, characterization and potential applications in chemical and biological sensors. ACS App Mater Inf 2009;1:1599-1606.

7. Sangodkar H, Sukeerthi S, Srinivasa RS, Lal R, Contractor AQ. A biosensor array based on polyaniline. Anal Chem 1996;68: 779-783.

8. MacDonald RA, Laurenzi BF, Viswanathan G, Ajayan PM, Stegemann JP. Collagen-carbon nanotube composite materials as scaffolds in tissue engineering. J Biomed Mater Res A 2005;74:489-496.

9. Crouzier T, Nimmagadda A, Nollert MU, McFetridge PS. Modification of single walled carbon nanotube surface chemistry to improve aqueous solubility and enhance cellular interactions. Langmuir 2008;24:13173-13181.

10. Keefer EW, Botterman BR, Romero MI, Rossi AF, Gross GW. Carbon nanotube coating improves neuronal recordings. Nat Nano 2008;3:434-439.

11. MacDonald RA, Voge CM, Kariolis M, Stegemann JP. Carbon nanotubes increase the electrical conductivity of fibroblast-seeded collagen hydrogels. Acta Biomater 2008;4:1583-1592.

12. Voge CM, Kariolis M, MacDonald RA, Stegemann JP. Directional conductivity in SWNT-collagen-fibrin composite biomaterials through strain-induced matrix alignment. J Biomed Mater Res A 2008;86:269-277.

13. Banerjee S, Hemraj-Benny $T$, Wong SS. Covalent surface chemistry of single-walled carbon nanotubes. Adv Mater 2005;17:17-29.

14. Peng $\mathrm{H}$, Alemany LB, Margrave JL, Khabashesku VN. Sidewall carboxylic acid functionalization of single-walled carbon nanotubes. J Am Chem Soc 2003;125:15174-15182.

15. Zhang J, Zou H, Qing Q, Yang Y, Li Q, Liu Z, Guo X, Du Z. Effect of chemical oxidation on the structure of single-walled carbon nanotubes. J Phys Chem B 2003;107:3712-3718.

16. Moore VC, Strano MS, Haroz EH, Hauge RH, Smalley RE. Individually suspended single-walled carbon nanotubes in various surfactants. Nano Lett 2003:3:1379-1382.
17. Wenseleers W, Vlasov II, Goovaerts E, Obraztsova ED, Lobach AS, Bouwen A. Efficient isolation and solubilization of pristine singlewalled nanotubes in bile salt micelles. Adv Funct Mater 2004;14: 1105-1112.

18. Ciofani G, Raffa V, Pensabene V, Menciassi A, Dario P. Dispersion of multi-walled carbon nanotubes in aqueous pluronic F127 solutions for biological applications. Fuller Nanotub Car N 2009;17:11.

19. Nativ-Roth E, Shvartzman-Cohen R, Bounioux C, Florent M, Zhang D, Szleifer I, Yerushalmi-Rozen R. Physical adsorption of block copolymers to SWNT and MWNT: A nonwrapping mechanism. Macromolecules 2007;40:3676-3685.

20. Schmolka IR. Artificial skin I. Preparation and properties of pluronic F-127 gels for treatment of burns. J Biomed Mater Res 1972; 6:571-582.

21. Dresselhaus MS, Dresselhaus G, Saito R, Jorio A. Raman spectroscopy of carbon nanotubes. Phys Reports 2005;409:47-99.

22. Hiura H. Raman studies of carbon nanotubes. Chem Phys Lett 1993;202:509-512.

23. Behler K, Osswald S, Ye H, Dimovski S, Gogotsi Y. Effect of thermal treatment on the structure of multi-walled carbon nanotubes. J Nanopart Res 2006;8:615-625.

24. George JJ, Sengupta R, Bhowmick AK. Influence of functionalization of multi-walled carbon nanotubes on the properties of ethylene vinyl acetate nanocomposites. J Nanosci Nanotechnol 2008;8: 1913-1921.

25. Sinani VA, Gheith MK, Yaroslavov AA, Rakhnyanskaya AA, Sun $K$ Mamedov AA, Wicksted JP, Kotov NA. Aqueous dispersions of single-wall and multiwall carbon nanotubes with designed amphiphilic polycations. JACS 2005;127:3463-3472.

26. Hu H, Yu A, Kim E, Zhao B, Itkis ME, Bekyarova E, Haddon RC. Influence of the zeta potential on the dispersability and purification of single-walled carbon nanotubes. J Phys Chem B 2005;109: $11520-11524$.

27. Magnusson G, Olsson T, Nyberg J-A. Toxicity of pluronic F-68. Toxicol Lett 1986;30:203-207.

29. Bardi G, Tognini P, Ciofani G, Raffa V, Costa M, Pizzorusso T. Pluronic-coated carbon nanotubes do not induce degeneration of cortical neurons in vivo and in vitro. Nanomed Nanotechnol 2009; 5:96-104.

29. Musumeci AW, Silva GG, Liu J-W, Martens WN, Waclawik ER. Structure and conductivity of multi-walled carbon nanotube/ poly(3-hexylthiophene) composite films. Polymer 2007;48: 1667-1678.

30. Sandler JKW, Kirk JE, Kinloch IA, Shaffer MSP, Windle AH. Ultralow electrical percolation threshold in carbon-nanotube-epoxy composites. Polymer 2003;44:5893-5899.

31. Li J, Ma PC, Chow WS, To CK, Tang BZ, Kim J-K. Correlations between percolation threshold, dispersion state and aspect ratio of carbon nanotubes. Adv Funct Mater 2007;17:3207-3215.

32. Quent VMC, Loessner D, Friis T, Reichert JC, Hutmacher DW. Discrepancies between metabolic activity and DNA content as tool to assess cell proliferation in cancer research. J Cell Mol Med 2010; 14:1003-1013.

33. Wörle-Knirsch JM, Pulskamp K, Krug HF. Oops they did it again! Carbon nanotubes hoax scientists in viability assays. Nano Lett 2006;6:1261-1268

34. Wick $P$, Manser $P$, Limbach LK, Dettlaff-Weglikowska U, Krumeich F, Roth S, Stark WJ, Bruinink A. The degree and kind of agglomeration affect carbon nanotube cytotoxicity. Toxicol Lett 2007;168: 121-131.

35. Nimmagadda A, Thurston K, Nollert MU, McFetridge PS. Chemical modification of SWNT alters in vitro cell-SWNT interactions. J Biomed Mater Res A 2006;76:614-625.

36. Belyanskaya L, Weigel $S$, Hirsch $C$, Tobler U, Krug HF, Wick P. Effects of carbon nanotubes on primary neurons and glial cells. NeuroToxicology 2009;30:702-711. 